\title{
On Types of Quasifree Representations of Clifford Algebras
}

\author{
By \\ Toshiki Murakami* and Shigeru Yamagami*
}

\begin{abstract}
The types of von Neumann algebras generated by quasifree representations of infinite dimensional Clifford algebras are studied in terms of spectral properties of positive operators parametrizing quasifree states.
\end{abstract}

\section{§1. Introduction}

The type analysis of quasifree factors (i.e., factors generated by quasifree representations) has been an interesting problem since the appearance of the pioneering work of Araki and Wyss [4]. In the case of CAR-algebras, the rough classification into type $\mathrm{I}_{\infty}, \mathrm{II}_{1}, \mathrm{II}_{\infty}$, and III was obtained in late 60's by several authors (see [6], [9] for example).

In this paper, we shall describe the fine classification of type III quasifree factors (i.e., factors arising from quasifree representations) in terms of spectral properties of positive operators parametrizing quasifree states (i.e., positive operators associated to two-point functions). Roughly speaking, the essense of our analysis goes back to the celebrated work by Araki and Woods on infinite tensor product factors but there are two points we should take notice of:

(i) At the starting point, we have no restrictions (such as discreteness) on the spectrum of positive operators $S$. Fortunately we can modify $S$ up to quasi-equivalence so that it has only point spectrum as was done in [6], [9], [10], [1]. The type analysis is achieved by studying this modified operator based on [3] and then relating it to the original $S$. In this last process, we need the duality between the $T$-set and the asymptotic ratio set (or more generally the Connes' $S$-set) established by A. Connes.

Communicated by H. Araki, May 27, 1993. Revised May 12, 1994.

1991 Mathematics Subject Classifications: 46L35, 46K10.

* Department of Mathematics, Tohoku University, Sendai 980, Japan. 
(ii) The approach described above is only valid when the dimension of the kernel of $S-1 / 2$ is even (this corresponds to the case of CAR-algebras). If $\operatorname{ker}(S-1 / 2)$ is odd-dimensional, we identify the quasifree factor $R_{S}$ associated to $S$ with a crossed product of a quasifree factor of even-dimensional case by an outer action of $\mathbb{Z}_{2}$. The type analysis is then done with the help of two general results on types of crossed product algebras due to [Sauvageot] and [Loi] respectively which especially make sense in the above situation.

We have clarified these two points in the present paper and obtained a necessary and sufficient condition for quasifree representations to generate type $\mathrm{III}_{\lambda}$-factors. Since the generic quasifree factors are known to be of type $\mathrm{III}_{1}$, this provides the fine classification.

The authors are grateful to $\mathrm{H}$. Kosaki and K. Saito for their interests in the present work which has been a great encouragement in preparing the paper.

\section{Notation}

For a self-adjoint operator $S$ in a Hilbert space and a subset $I$ of the real line, we denote by $S_{I}$ the self-adjoint operator cut down to the spectral subspace corresponding to $I$. When $I$ consists of one point, say $a$, we write as $S_{a}$ instead of $S_{\{a\}}$.

A self-adjoint operator $S$ is said to have pure point spectrum if the spectral measure associated to $S$ is supported by a countable (not necessarily closed) subset of $\mathbf{R}$.

\section{§ 2. Preliminaries}

In this section we collect together some of basic facts on quasifree representations of Clifford $C^{*}$-algebras.

Let $H$ be a separable infinite dimensional Hilbert space with complex conjugation - (i.e., ${ }^{-}$is a conjugate-linear involution satisfying $\langle\bar{f}, \bar{g}\rangle=\langle g, f\rangle$ ).

We consider a *-algebra $C_{0}(H)$ generated by elements in $H$ and the unit 1 with the relations

$$
f^{*}=\bar{f}, \quad f^{*} g+g f^{*}=\langle f, g\rangle 1 \quad(f, g \in H) .
$$

It is well known that the *-algebra $C_{0}(H)$ is isomorphic to an inductive limit of matrix algebras of the form $M_{2^{d}}(\mathrm{C})$ (consider a directed system indexed by finite-dimensional subspaces of $H$ ). In particular, there is the unique $C^{*}$-norm under which $C_{0}(H)$ is completed to a $C^{*}$-algebra $C(H)$, called the Clifford $C^{*}$-algebra. Let $U$ be an orthogonal transformation in $H$, i.e., a unitary operator in $H$ which commutes with the conjugation - . As a consequence of the universality of construction, $U$ is uniquely extended to a $*$-automorphism $\tau(U)$ of $C(H)$, called a Bogoliubov automorphism according to Araki. 
For $f \in H$, its $C^{*}$-norm is calculated by the formula (see [2])

$$
\|f\|_{\mathbf{C}^{*}}=2^{-1 / 2}\left(\|f\|^{2}+\left(\|f\|^{4}-|\langle f, \bar{f}\rangle|^{2}\right)^{1 / 2}\right)^{1 / 2} .
$$

In particular the imbedding $H \hookrightarrow C(H)$ is continuous. Given a state $\varphi$ of $C(H)$, the two-point function of $\varphi$ is, by definition, a positive hermitian form $\varphi\left(f^{*} g\right)$ on $H$, which is continuous with respect to the Hilbert space norm. Hence we obtain a (bounded) positive linear operator $S$ in $H$ such that $\varphi\left(f^{*} g\right)=\langle f, S g\rangle$. From the commutation relation (1), the operator $S$ satisfies

$$
S+\bar{S}=1
$$

Here $\bar{S}$ denotes the complex conjugation of $S$ relative to ${ }^{-}$, i.e., $\bar{S}={ }^{-} \circ S^{-}{ }^{-}$.

Generally the operator $S$ is only a part of information of states. There is, however, a canonical way to associate states to such operators ([1]): Given a positive operator $S$ fulfilling (3), there is the (unique) state $\varphi_{S}$, called quasifree state, determined by

$$
\begin{aligned}
\varphi_{S}\left(f_{1} f_{2} \cdots f_{2 n+1}\right) & =0, \\
\varphi_{S}\left(f_{1} f_{2} \cdots f_{2 n}\right) & =\sum \operatorname{sgn}(\sigma) \varphi_{S}\left(f_{\sigma(1)} f_{\sigma(2)}\right) \cdots \varphi_{S}\left(f_{\sigma(2 n-1)} f_{\sigma(2 n)}\right),
\end{aligned}
$$

where the summation is taken over permutations of $\{1, \ldots, 2 n\}$ such that

$$
\sigma(1)<\sigma(3)<\cdots<\sigma(2 n-1) \text { and } \sigma(2 j-1)<\sigma(2 j) \quad \text { for } j=1, \ldots, n \text {. }
$$

We denote by $\pi_{S}$ the GNS-representation of $C(H)$ associated to the quasifree state $\varphi_{S}$ and by $R_{S}$ the von Neumann algebra generated by the image of $\pi_{s}$.

We list some of basic properties of quasifree representations.

Theorem 2.1 ([1], [9]). Let $S, T$ be positive operators in $B(H)$ which satisfy (3). Then two representations $\pi_{S}$ and $\pi_{T}$ are quasi-equivalent if and only if $S^{1 / 2}-T^{1 / 2}$ is a Hilbert-Schmidt operator.

Note here that a Bogoliubov automorphism $\tau(U)$ is extended to an automorphism of $R_{S}$ if and only if $U S^{1 / 2} U^{-1}-S^{1 / 2}$ is in the Hilbert-Schmidt class and, if this is the case, the extended automorphism of the von Neumann algebra is also denoted by $\tau_{S}(U)$. The following fact can easily be checked with the help of the KMS-condition.

Lemma 2.2. The modular automorphism group $\left\{\sigma_{t}^{\varphi_{S}}\right\}_{t \in \mathbf{R}}$ of the quasifree state $\varphi_{S}$ on $R_{S}$ is given in the form of Bogoliubov automorphism:

$$
\sigma_{t}^{\varphi_{S}}=\tau_{S}\left(S^{i t}(1-S)^{-i t}\right) .
$$

The condition for $R_{S}$ to be a factor is considered in [1], [9]. 
Theorem 2.3 ([1], [9]). If the kernel of $S-1 / 2$ is even-dimensional (including dimensions 0 and $\infty$ ), then $R_{S}$ is a factor. When the kernel of $S-1 / 2$ is (finite) odd-dimensional, then $R_{S}$ is not a factor if and only if $S_{[0,1 / 2]}$ is a traceclass operator. If this is the case, $R_{S}$ is isomorphic to $B(K) \oplus B(K)$ with $K$ an infinite dimensional Hilbert space.

Theorem 2.4 ([8], [9]). Suppose that the kernel of $S-1 / 2$ is even-dimensional. Then the following holds.

(i) $R_{S}$ is a $I_{\infty}$-factor if and only if $S_{[0,1 / 2]}$ is a trace class operator.

(ii) $R_{S}$ is a $\mathrm{II}_{1}$-factor if and only if $S^{1 / 2}-(1 / 2)^{1 / 2}$ is a Hilbert-Schmidt operator.

(iii) $R_{S}$ is a $\mathrm{II}_{\infty}$-factor if and only if $S$ is a combination of (1) and (2), i.e., $S_{[0, c]}$ is a trace class operator and $S_{[c, 1 / 2]}^{1 / 2}-(1 / 2)^{1 / 2}$ is a HilbertSchmidt operator for some and hence any $0<c<1 / 2$ with both of these operators having infinite-dimensional ranges.

(iv) $R_{S}$ is a III-factor if $S$ does not satisfy any of the above three conditions.

The following is obtained by modifying the proof of von Neumann's lemma on Hilbert-Schmidt perturbation of self-adjoint operators (cf. [9, Lemma 4.3]).

Theorem 2.5. Let $S$ be an operator in $B(H)$ satisfying (3). Then there exists a positive operator $T$ in $B(H)$ with pure point spectrum and satisfying (3) such that $S^{1 / 2}-T^{1 / 2}$ is a Hilbert-Schmidt operator. For any such a perturbation, the parity of $\operatorname{dim} \operatorname{ker}(S-1 / 2)$ is unchanged.

We need the following criterion for the innerness of product type automorphisms.

Lemma 2.6 ([5]). Let $\left\{\varphi_{j}\right\}_{j \geq 1}$ be a family of states on the $2 \times 2$ matrix algebra $M_{2}(\mathbb{C})$ and $\left\{u_{j}\right\}_{j \geq 1}$ be a family of unitary matrices in $M_{2}(\mathbf{C})$ such that $\varphi_{j}$ commutes with $u_{j}$ for all $j \geq 1$ (i.e., if we express $\varphi_{j}$ as $\varphi_{j}(\cdot)=\operatorname{tr}\left(\rho_{j} \cdot\right)$ with $\rho_{j}$ a positive matrix, then $\rho_{j} u_{j}=u_{j} \rho_{j}$ for $j \geq 1$ ). Then the automorphism $\bigotimes_{j \geq 1} A d u_{j}$ in the infinite tensor product factor $\bigotimes_{j \geq 1}\left(M_{2}(\mathbb{C}), \varphi_{j}\right)$ is inner iff

$$
\sum_{j \geq 1}\left(1-\left|\varphi_{j}\left(u_{j}\right)\right|\right)<+\infty
$$

If this condition is satisfied, a unitary operator implementing this inner automorphism is given by $\bigotimes_{j \geq 1} e^{i \theta_{j}} u_{j}$, where $e^{i \theta_{j}}$ is a phase factor determined by $e^{i \theta_{j}} \varphi_{j}\left(u_{j}\right)>0$.

For later use, we rewrite this result into the following form. Suppose that a positive operator $S$ satisfying (3) has pure point spectrum and the kernel of $S-1 / 2$ is even-dimensional. Then $S$ is decomposed as 


$$
S=\sum_{j=1}^{\infty}\left\{\xi_{j} E_{j}+\left(1-\xi_{j}\right) \bar{E}_{j}\right\}
$$

where $0 \leq \xi_{j} \leq 1 / 2$ is the half of eigenvalues of $S$ and $E_{j}(j=1,2, \ldots)$ denote mutually orthogonal one-dimensional projections.

Corollary 2.7. If $S$ takes the form in equation (6) and $\operatorname{dim}(\operatorname{ker}(1 / 2-S))$ is even, then the modular automorphism $\sigma_{t}^{\varphi_{S}}(t \in \mathbf{R})$ is inner if and only if

$$
\sum_{i=1}^{\infty} \xi_{j}\left(1-\cos \left[t\left(\log \xi_{j}-\log \left(1-\xi_{j}\right)\right)\right]\right)<\infty .
$$

Proof. This is definitely well-known but for the sake of completeness, we recall the points of arguments. Let $f_{j}, \bar{f}_{j}(j \geq 1)$ be an orthogonal basis corresponding to the decomposition $1=\sum_{j \geq 1}\left(E_{j}+\bar{E}_{j}\right)\left(f_{j} \in E_{j} H\right)$. Then we can define a family of mutually commuting $2 \times 2$-matrix units $\left\{e_{i j}^{(n)}\right\}_{n \geq 1}$ by

$$
\begin{array}{ll}
e_{11}^{(n)}=f_{n} f_{n}^{*}, & e_{12}^{(n)}=f_{n} \prod_{r=1}^{n-1}\left(1-2 f_{r}^{*} f_{r}\right), \\
e_{21}^{(n)}=f_{n}^{*} \prod_{r=1}^{n-1}\left(1-2 f_{r}^{*} f_{r}\right), & e_{22}^{(n)}=f_{n}^{*} f_{n}
\end{array}
$$

and $C(H)$ is identified with the infinite product $C^{*}$-algebra $\otimes_{n \geq 1} M_{2}(\mathbf{C})$. With this identification, the quasifree state $\varphi_{S}$ is regarded as the product state $\otimes_{j \geq 1} \varphi_{j}$ with

$$
\varphi_{i}(\cdot)=\operatorname{tr}\left(\left(\begin{array}{cc}
1-\xi_{i} & 0 \\
0 & \xi_{i}
\end{array}\right) \cdot\right)
$$

and the modular automorphism $\bigotimes_{j \geq 1} A d\left(\begin{array}{cc}1-\xi_{i} & 0 \\ 0 & \xi_{i}\end{array}\right)^{i t}$.

Now applying Lemma 2.6, one sees that $t \in \mathbf{R}$ is in the $T$-set for $R_{S}$ if and only if

$$
\sum_{j \geq 1}\left(1-\left|1-\xi_{j}+\xi_{j}^{i t}\left(1-\xi_{j}\right)^{-i t} \xi_{j}\right|\right)<+\infty
$$

By a direct computation of the absolute value of $1-\xi_{j}+\xi_{j}^{i t}\left(1-\xi_{j}\right)^{-i t} \xi_{j}$, the summand in the above condition takes the form $1-\sqrt{1-2 \xi_{j}\left(1-\xi_{j}\right)\left(1-\cos \theta_{j}\right)}$ with $\theta_{j}=t\left(\log \xi_{j}-\log \left(1-\xi_{j}\right)\right)$. The assertion is then obtained from $1-x \leq$ $\sqrt{1-x} \leq 1-x / 2$ for $0 \leq x \leq 1$ (the factor $1-\xi_{j}$ is omitted because $0 \leq$ $\left.\xi_{j} \leq 1 / 2\right)$.

Corollary 2.8. Let $S$ be a positive operator which satisfies (3) and has pure point spectrum. Let $U \in B(H)$ be a unitary operator which can be diagonalized in the following form:

$$
U=\sum_{j=1}^{\infty} u_{j} E_{j}+\bar{u}_{j} \bar{E}_{j}
$$


(Noted that $U$ commutes with $S$ and $u_{j}^{\prime}$ s are complex numbers of modulus 1.) Then the Bogoliubov automorphism $\tau(U)$ of $R_{S}$ is inner if and only if

$$
\sum_{j=1}^{\infty}\left(1-\left|\left(u_{i}-1\right) \xi_{i}+1\right|\right)<+\infty .
$$

Proof. With the same notation in the proof of Corollary 2.7, the Bogoliubov automorphism $\tau_{S}(U)$ is identified with

$$
\bigotimes_{j \geq 1} A d\left(\begin{array}{ll}
u_{j} & 0 \\
0 & 1
\end{array}\right)
$$

and we can apply Lemma 2.6 again.

Since the quasifree state $\varphi_{S}$ with $S$ given by (6) is a product state, we can talk about the asymptotic ratio set of $\varphi_{S}$. Recall the definition of asymptotic ratio set in our context (see [3] for the original definition): Let $\xi=\left\{\xi_{j}\right\}_{j \geq 1}$ be a sequence of positive numbers with $\xi_{j} \leq 1 / 2$ for $j \geq 1$. For a finite set $I$ consisting of positive integers, let $\{0,1\}^{I}$ be the set of functions on $I$ with values in $\{0,1\}$. For an element $\varepsilon$ in $\{0,1\}^{I}$, set

$$
\xi(\varepsilon)=\prod_{j \in I} \xi_{j}^{(\varepsilon(j))},
$$

where $\xi_{j}^{(0)}=1-\xi_{j}$ and $\xi_{j}^{(1)}=\xi_{j}$ and, for a subset $E \subset\{0,1\}^{I}$, define $\xi(E)$ by

$$
\xi(E)=\sum_{\varepsilon \in E} \xi(\varepsilon)
$$

The asymptotic ratio set $r_{\infty}$ of $\xi$ (or $R_{S}$ if $\xi$ is the sequence in (6)) is the totality of non-negative real numbers $r$ such that there is a sequence of mutually disjoint finite sets $I_{n}$ consisting of positive integers, a sequence of subsets $E_{n} \subset I_{n}$, and a sequence of injective mappings $\phi_{n}: E_{n} \rightarrow\{0,1\}^{I_{n}} \backslash E_{n}$ with the properties

$$
\sum_{n=1}^{\infty} \xi\left(E_{n}\right)=\infty
$$

and

$$
\lim _{n \rightarrow \infty} \max _{\varepsilon \in E_{n}}\left|r-\frac{\xi\left(\phi_{n}(\varepsilon)\right)}{\xi(\varepsilon)}\right|=0 .
$$

Note that accumulation points of the sequence $\left\{\xi_{j} /\left(1-\xi_{j}\right)\right\}_{j \geq 1}$ are contained in the asymptotic ratio set of $R_{S}$ except for 0 . From this simple observation, we have the following ([1]):

Lemma 2.9. Suppose that $S$ is given by (6).

(i) If the sequence $\left\{\xi_{j}\right\}_{j \geq 1}$ has (at least) two accumulation points $\{\lambda /(1+\lambda)$, $\mu /(1+\mu)\}(0<\lambda, \mu<1)$ such that $\lambda$ is not a rational power of $\mu$, then $R_{S}$ is a $\mathrm{III}_{1}$-factor. 
(ii) If $R_{S}$ is of type $\mathrm{III}_{0}$, the accumulation points of the sequence $\left\{\xi_{j}\right\}_{j \geq 1}$ are contained in the set $\{0,1 / 2\}$.

\section{§3. Type Analysis-even-dimensional case}

In this section we assume that $\operatorname{dim}(\operatorname{ker}(1 / 2-S))$ is even.

Definition 3.1. Let $0<\lambda<1$. A sequence $\left\{n_{j}\right\}_{j \geq 1}$ of positive integers is called a $\lambda$-sequence if there exist a disjoint sequence $\left\{I_{n}\right\}$ of finite subsets in $\mathbf{N}$, a sequence of subsets $E_{n} \subset\{0,1\}^{I_{n}}$, and a sequence of injective mappings $\phi_{n}: E_{n} \rightarrow\{0,1\}^{I_{n}} \backslash E_{n}$ such that

$$
\begin{aligned}
\sum_{n \geq 1} \lambda\left(E_{n}\right) & =+\infty, \\
\left|\phi_{n}(\varepsilon)\right|-|\varepsilon| & =1 \quad \text { for } \forall n \geq 1, \quad \forall \varepsilon \in E_{n} .
\end{aligned}
$$

Here $|\varepsilon|=\sum_{\varepsilon(j)=1} n_{j}$ (the summation is taken over $j \in I_{n}$ satisfying $\varepsilon(j)=1$ ) and

$$
\lambda(E)=\sum_{\varepsilon \in E} \frac{\lambda^{|\varepsilon|}}{\prod_{j \in I}\left(1+\lambda^{n_{j}}\right)} .
$$

Lemma 3.2. Assume that $\xi_{j}=\lambda^{n_{j}} /\left(1+\lambda^{n_{j}}\right)$ with $\left\{n_{j}\right\}$ a sequence of nonnegative integers. Then $R_{S}$ is of type $\mathrm{III}_{\lambda}$ if and only if $\left\{n_{j}\right\}$ is a $\lambda$-sequence.

Proof. This is just a restatement of the definition.

Theorem 3.3. Suppose that $\operatorname{dim}(\operatorname{ker}(1 / 2-S))$ is even. For $0<\lambda<1, R_{S}$ is a $\mathrm{III}_{\lambda}$-factor if and only if the following holds:

(i) The spectrum of $S$ is discrete.

(ii) Let $\left\{E_{j}\right\}_{j \geq 1}$ be a mutually orthogonal sequence of spectral projections of rank 1 for $S$ with $0 \leq \xi_{j} \leq 1 / 2$ the corresponding eigenvalues. Then there is a $\lambda$-sequence $\left\{n_{j}\right\}_{j \geq 1}$ such that

$$
\sum_{j=1}^{\infty}\left|\xi_{j}^{1 / 2}-\left(\frac{\lambda^{n_{j}}}{1+\lambda^{n_{j}}}\right)^{1 / 2}\right|^{2}<+\infty .
$$

Proof. By Lemma 3.1, the conditions in (i) and (ii) are sufficient to insure that $R_{S}$ is of type III $_{\lambda}$.

Conversely assume that $R_{S}$ is a $\mathrm{III}_{\lambda}$-factor. Let $T$ be an operator in Theorem 2.5. By Theorem 2.1, $R_{S}$ and $R_{T}$ are isomorphic and hence $R_{T}$ is of type III $_{\lambda}$. Then the accumulation points of the spectrum (counting multiplicity) are contained in the set $\{0\} \cup\left\{\lambda^{n} /\left(1+\lambda^{n}\right) ; n \in \mathbf{Z}\right\}$. Since the essential spectra of $S$ and $T$ coincide, this implies that the spectrum of $S$ is discrete and accumulates at most in $\{0\} \cup\left\{\lambda^{n} /\left(1+\lambda^{n}\right), 1 /\left(1+\lambda^{n}\right) ; n=0,1,2, \ldots\right\}$. In particular, we have the expression for $S$ as in (6). 
For each $j \geq 1$, define an integer $n_{j}$ and a real number $-1 / 2<\alpha_{j} \leq 1 / 2$ by $\xi_{j} /\left(1-\xi_{j}\right)=\lambda^{n_{j}+\alpha_{j}}$. Since $2 \pi / \lambda$ is in the $T$-set of $R_{S}$, Corollary 2.7 shows that

$$
\sum_{j \geq 1} \xi_{j}\left(\sin \pi \frac{\log \xi_{j}-\log \left(1-\xi_{j}\right)}{\log \lambda}\right)^{2}<+\infty
$$

i.e.,

$$
\sum_{j \geq 1} \lambda^{n_{j}}\left(\sin \pi \alpha_{j}\right)^{2}<+\infty
$$

Since $|2 \theta / \pi| \leq|\sin \theta| \leq|\theta|$ for $-\pi / 2 \leq \theta \leq \pi / 2$, this is further equivalent to

$$
\sum_{j=1}^{\infty} \lambda^{n_{j}} \alpha_{j}^{2}<+\infty
$$

Now define $S_{0} \in B(H)$ by

$$
S_{0}=\sum_{j=1}^{\infty}\left(\frac{\lambda^{n_{j}}}{1+\lambda^{n_{j}}} E_{j}+\frac{1}{1+\lambda^{n_{j}}} \bar{E}_{j}\right) .
$$

The positive operator $S_{0}$ satisfies (3) and we can show that

$$
\begin{aligned}
\left\|S^{1 / 2}-S_{0}^{1 / 2}\right\|_{H . S .}^{2}= & \sum_{j=1}^{\infty}\left(\left(\frac{\lambda^{n_{j}+\alpha_{j}}}{1+\lambda^{n_{j}+\alpha_{j}}}\right)^{1 / 2}-\left(\frac{\lambda^{n_{j}}}{1+\lambda^{n_{j}}}\right)^{1 / 2}\right)^{2} \\
& +\sum_{j=1}^{\infty}\left(\left(\frac{1}{1+\lambda^{n_{j}+\alpha_{j}}}\right)^{1 / 2}-\left(\frac{1}{1+\lambda^{n_{j}}}\right)^{1 / 2}\right)^{2}
\end{aligned}
$$

converges. In fact, for the convergence of the first summation, we deduce as follows:

$$
\begin{aligned}
& \sum_{j \geq 1}\left(\left(\frac{\lambda^{n_{j}+\alpha_{j}}}{1+\lambda^{n_{j}+\alpha_{j}}}\right)^{1 / 2}-\left(\frac{\lambda^{n_{j}}}{1+\lambda^{n_{j}}}\right)^{1 / 2}\right)^{2} \\
& \quad=\sum_{j \geq 1} \lambda^{n_{j}}\left(\left(\lambda^{\alpha_{j}}+\lambda^{n_{j}+\alpha_{j}}\right)^{1 / 2}-\left(1+\lambda^{n_{j}+\alpha_{j}}\right)^{1 / 2}\right)^{2}\left(1+\lambda^{n_{j}}\right)^{-1}\left(1+\lambda^{n_{j}+\alpha_{j}}\right)^{-1} \\
& \quad \sim \sum_{j \geq 1} \lambda^{n_{j}}\left(\left(\lambda^{\alpha_{j}}+\lambda^{n_{j}+\alpha_{j}}\right)^{1 / 2}-\left(1+\lambda^{n_{j}+\alpha_{j}}\right)^{1 / 2}\right)^{2} \\
& \quad \sim \sum_{j \geq 1} \lambda^{n_{j}}\left(\left(\lambda^{\alpha_{j}}+\lambda^{n_{j}+\alpha_{j}}\right)-\left(1+\lambda^{n_{j}+\alpha_{j}}\right)\right)^{2} \\
& \quad=\sum_{j \geq 1} \lambda^{n_{j}}\left(\lambda^{\alpha_{j}}-1\right)^{2} .
\end{aligned}
$$

(Here $\sum_{j} a_{j} \sim \sum_{j} b_{j}$ means that $\sum_{j} a_{j}<+\infty$ if and only if $\sum_{j} b_{j}<+\infty$.) Since $\left(\lambda^{x}-1\right)^{2} \leq \lambda^{-1}|\log \lambda| x^{2}$ for $-1 / 2 \leq x \leq 1 / 2$, the last summation converges due to (10). (We have in fact showed that the summation in (ii) converges for $\left\{n_{j}\right\}$ just defined.) Similarly for the second summation. 
In this way, we showed that $\varphi_{S}$ and $\varphi_{S_{0}}$ are quasi-equivalent. Then from the assumption, $R_{S_{0}}$ is of type III $_{\lambda}$. Since the half of the spectrum of $S_{0}$ is given by the sequence $\left\{\lambda^{n_{j}} /\left(1+\lambda^{n_{j}}\right)\right\}_{j \geq 1},\left\{n_{j}\right\}$ is a $\lambda$-sequence by Lemma 3.1.

Corollary 3.4. If $S$ is invertible, then $R_{S}$ is a $\mathrm{III}_{\lambda}$-factor with $0<\lambda<1$ if and only if

(i) the spectrum of $S$ is discrete and

(ii) with the same notation as in the theorem (ii), there is a bounded sequence $\left\{n_{j}\right\}$ of non-negative integers such that the greatest common divisor of the values which appear in the sequence with multiplicity infinite is equal to 1 and

$$
\sum_{j \geq 1}\left|\xi_{j}^{1 / 2}-\left(\frac{\lambda^{n_{j}}}{1+\lambda^{n_{j}}}\right)^{1 / 2}\right|^{2}<+\infty .
$$

Proof. Suppose that $R_{S}$ is of type $\mathrm{III}_{\lambda}$ and let $\left\{n_{j}\right\}$ be a $\lambda$-sequence which is assured in the theorem. Since $S$ is assumed to be invertible, the sequence $\left\{n_{j}\right\}$ is bounded. Let $\left\{m_{1}, \ldots, m_{d}\right\}$ be the set of values which appear in $\left\{n_{j}\right\}$ infinitely many times. Let $m$ be the common divisor of $\left\{m_{1}, \ldots, m_{d}\right\}$. Since the values of finite multiplicity have no effect on the type of generated von Neumann algebras, the asymptotic ratio set is contained in the set of integerpowers of $\lambda^{m}$. Thus $m=1$.

Conversely suppose that we can find a sequence of integers $\left\{l_{1}, \ldots, l_{d}\right\}$ such that $l_{1} m_{1}+\cdots+l_{d} m_{d}=1$. We may assume that $l_{1}>0, \ldots, l_{k}>0, l_{k+1}<$ $0, \ldots, l_{k+f}<0$, and $l_{k+f+1}=\cdots=l_{d}=0$. Since each $m_{j}$ is of infinite multiplicity, we can find a disjoint sequence $\left\{I_{n}\right\}$ of sets consisting of positive integers such that $m_{j}$ appears in $I_{n}$ with multiplicity $\left|l_{j}\right|$. For each $n$, define an element $\varepsilon_{n} \in\{0,1\}^{I_{n}}$ by

$$
\varepsilon_{n}(j)= \begin{cases}0 & \text { if } n_{j} \text { is in }\left\{m_{1}, \ldots, m_{k}\right\} \\ 1 & \text { otherwise }\end{cases}
$$

Let $E_{n}=\left\{\varepsilon_{n}\right\}$ and define $\phi_{n}: E_{n} \rightarrow\{0,1\}^{I_{n}} \backslash E_{n}$ by

$$
\phi_{n}\left(\varepsilon_{n}\right)(j)= \begin{cases}1 & \text { if } n_{j} \text { is in }\left\{m_{k+1}, \ldots, m_{d}\right\} \\ 0 & \text { otherwise }\end{cases}
$$

Then $\left|\phi_{n}\left(\varepsilon_{n}\right)\right|-\left|\varepsilon_{n}\right|=1$ while the summation of

$$
\lambda\left(E_{n}\right)=\lambda^{l_{1} m_{1}+\cdots+l_{k} m_{k}} / \prod_{j \in I_{n}}\left(1+\lambda^{n_{j}}\right)=\lambda / \prod_{1 \leq j \leq d}\left(1+\lambda^{m_{j}}\right)^{\left|l_{j}\right|}
$$

for $n \geq 1$ diverges. Thus $\left\{n_{j}\right\}$ is a $\lambda$-sequence. 


\section{§4. Type Analysis - odd-dimensional case}

In this section we exclusively deal with the case $\operatorname{ker}(S-1 / 2)$ being odddimensional. In that case we can find a real (i.e., $\bar{f}=f$ ) normalized vector $f$ in $\operatorname{ker}(S-1 / 2)$ and, if we let $S_{0}$ be the restriction of $S$ to the orthogonal complement $H \ominus \mathbb{C} f$, then $\operatorname{ker}\left(S_{0}-1 / 2\right)$ is even-dimensional. The unitary $u=$ $\pi_{S}(\sqrt{2} f)$ in $R_{S}$ is self-adjoint and implements the Bogoliubov automorphism $\tau(-1)$ on $R_{S_{0}}$. Thus $R_{S}$ is identified with the crossed product algebra $R_{S_{0}} \times$ $\mathbb{Z}_{2}$. Note that $\tau(-1)$ is outer if $R_{S}$ is a factor (if $\tau(-1)=A d v$ on $R_{S_{0}}$ with $v \in R_{S_{0}}$, then $u^{-1} v$ is in the center of $R_{S}$ ).

Since the classification of von Neumann algebras into types $\mathbb{I}, \mathbb{I I}_{1}, \mathbb{I}_{\infty}$, or III is preserved under the crossed product by finite groups (cf. [13], §22.7), we have

Theorem 4.1 ([8], [9]). Suppose that the kernel of $S-1 / 2$ is odd-dimensional. Then the following holds.

(i) $R_{S}$ is a direct sum of two $\mathrm{I}_{\infty}$-factors if and only if $S_{[0,1 / 2]}$ is a trace class operator.

(ii) $R_{S}$ is a $\mathrm{II}_{1}$-factor if and only if $S^{1 / 2}-(1 / 2)^{1 / 2}$ is a Hilbert-Schmidt operator.

(iii) $R_{S}$ is a $\mathrm{II}_{\infty}$-factor if and only if $S$ is a combination of (1) and (2), i.e., $S_{[0, c]}$ is a trace class operator and $S_{[c, 1 / 2]}^{1 / 2}-(1 / 2)^{1 / 2}$ is a HilbertSchmidt operator for some and hence any $0<c<1 / 2$ with both of these operators having infinite-dimensional ranges.

(iv) $R_{S}$ is a III-factor if $S$ does not satisfy any of the above three conditions.

Since $\varphi_{S}$ is invariant under $A d u$, we have the following (cf. [11], [12]).

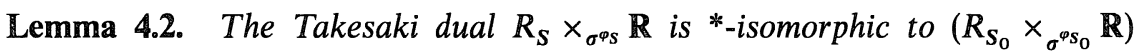
$\times \mathbb{Z}_{2}$ where the $\mathbb{Z}_{2}$-action on $R_{S_{0}} \times \mathbf{R}$ is defined as the extension of $\tau(-1)$ so that it fixes point-wise the 1-parameter group of unitaries implementing $\sigma_{t}^{\varphi_{S_{0}}}$.

For the description of $T$-sets of crossed products, we recall the result due to Sauvageot.

Proposition 4.3 [11, Proposition 3.9]. Let $M$ be a factor and $\left\{\alpha_{g}: g \in G\right\}$ be a discrete abelian automorphism group on $M$. Let $\varphi$ be a state on $M$ and $t \in R$. Then the following conditions are equivalent:

(i) $t \in T\left(M \times{ }_{\alpha} G\right)$.

(ii) There exist an element $g_{0} \in G$ and a unitary operator $v$ in $M$ such that

$$
\sigma_{t}^{\varphi} \circ \alpha_{g_{0}}=A d(v)
$$


and

$$
\left(D \varphi \circ \alpha_{g}: D \varphi\right)=\alpha_{g}(v) v^{*} \quad(g \in G) .
$$

Here we calculate the $T$-set of $R_{S}$.

\section{Lemma 4.4.}

$$
T\left(R_{S}\right)=T\left(R_{S_{0}}\right) \cup\left\{t \in \mathbf{R} ; \tau(-1) \sigma_{t}^{\varphi_{S_{0}}} \text { is inner }\right\} .
$$

Proof. Since $\varphi_{S}$ is $\tau(-1)$-invariant, we need to check that, when $\sigma_{t}$ or $\tau(-1) \sigma_{t}$ is inner, their implementing unitaries are fixed under $\tau(-1)$, which follows from the fact that the implementing operators are limits of even elements in $R_{S_{0}}$ as seen in Lemma 2.6.

We also need the following stability theorem of types under crossed products by finite groups, which would be well-known. Since it is contained in the Loi's work [7], we just cite it here (when the acting groups are abelian, the Takesaki's duality gives a simple proof).

Lemma 4.5. Let $N$ be a type III-factor and $G$ be a finite group. Let $\alpha: G \rightarrow$ Aut $(N)$ be an outer action. Then $N \times G$ is type $\mathrm{III}_{0}$ (resp. type $\mathrm{III}_{1}$ ) if and only if the same holds for $N$. If $N$ is of type $\mathrm{III}_{\lambda}$ with $0<\lambda<1$, then $N \times G$ is of type $\mathrm{III}_{\lambda q}$ with $q$ a positive rational number.

Lemma 4.6. Suppose that $R_{S_{0}}$ is a $\mathrm{III}_{\lambda}$-factor with $0<\lambda<1$ and let $\left\{n_{j}\right\}_{j \geq 1}$ be the associated $\lambda$-sequence in Theorem 3.3. Then we have

$$
T\left(R_{S}\right)= \begin{cases}\{n \pi / \log \lambda ; n \in \mathbb{Z}\} & \text { if } \sum \lambda^{n_{J}}<+\infty \\ \{2 n \pi / \log \lambda ; n \in \mathbb{Z}\} & \text { otherwise }\end{cases}
$$

Here the sum in the if-part is taken all $j$ such that $n_{j}$ is even.

Proof. Suppose that $\tau(-1) \sigma_{t}$ is inner on $R_{S_{0}}$. Since $\tau(-1)$ and $\sigma_{t}$ commute, $\sigma_{2 t}=\tau(-1) \sigma_{t} \tau(-1) \sigma_{t}$ is inner as well. Hence Lemma 4.4 shows that $T\left(R_{S}\right)=T\left(R_{S_{0}}\right)$ or $T\left(R_{S_{0}}\right) / 2$, the latter case occurs if and only if $\tau(-1) \sigma_{t}$ is inner for $t=\pi / \log \lambda$. By Corollary 2.8, this is equivalent to

$$
\sum_{j}\left(1-\left|1-\xi_{j}-\xi_{j}^{i t}\left(1-\xi_{j}\right)^{-i t} \xi_{j}\right|\right)<+\infty .
$$

Since $\xi_{j}=\lambda^{n_{J}} /\left(1+\lambda^{n_{j}}\right)$, the summation in the left hand side is given by

$$
\sum_{j}\left(1-\frac{1-(-1)^{n_{j}} \lambda^{n_{j}}}{1+\lambda^{n_{j}}}\right)=\sum_{n_{j}=\text { even }} \frac{2 \lambda^{n_{j}}}{1+\lambda^{n_{j}}},
$$

proving the assertion.

By Lemma 4.5 and Lemma 4.6, we finally have the following: 
Theorem 4.7. Suppose that $\boldsymbol{R}_{\boldsymbol{S}_{0}}$ is a $\operatorname{III}_{\lambda}$-factor $(0<\lambda<1)$ with $\left\{n_{j}\right\}_{j \geq 1}$ the $\lambda$-sequence assured in Theorem 3.3. Then $R_{S}$ remains to be a $\mathrm{III}_{\lambda}$-factor if

$$
\sum_{n_{j}: \text { even }} \lambda^{n_{j}}=+\infty
$$

Otherwise $R_{S}$ is a $\mathrm{III}_{\lambda^{2}}$-factor.

\section{References}

[1] Araki, H., On quasi-free states of CAR and Bogoliubov automorphisms, Publ. RIMS. Kyoto Univ., 6 (1970/71), 385-442.

[2] — Bogoliubov automorphisms and Fock representation of canonical anticommutation relation, Contemp. Math., 62 (1987), 23-141.

[3] Araki, H. and Woods, E. J., A classification of factors, Publ. RIMS. Kyoto Univ., 3 (1968), 51-130.

[4] Araki, H. and Wyss, W., Representations of canonical anticommutation relations, Helv. Phys. Acta, 37 (1964), 136-159.

[5] Connes, A., Une classification des facteurs de type III, Ann. Sci. Ec. Norm. Sup., 6 (1973), 133-252.

[6] Dell'Antonio, G. F., Structure of algebras of some free systems, Comm. Math. Phys., 9 (1968), 81-117.

[7] Loi, P. H., Sur la théorie de l'indice et les facteurs de type III, C.R. Acad. Sci. Paris, 305 (1987), 423-426.

[8] Moore, C. C., Invariant measures on product spaces, Proc. Fifth Berkley symposium on math, Vol II-Part II (1967), 447-459.

[9] Powers, R. T. and Størmer, E., Free states of canonical anticommutation relations, Comm. Math. Phys., 16 (1970), 1-33.

[10] Rideau, G., On some representations of anticommutation relations, Comm. Math. Phys., 9 (1968), 229-241.

[11] Sauvageot, J.-L., Sur le type du produit croise d'une algebre de von Neumann par un group localement compact, Bull. Soc. Math. France., 105 (1977), 349-368.

[12] Sekine, Y., Flow of weight of crossed product of type III factors by discrete groups, Publ. RIMS, Kyoto Univ., 26 (1990), 655-666.

[13] Strătilă, S., Modular Theory in Operator Algebras, Abacus Press, 1981. 